\title{
Genetics pathogenesis in the ovarian insufficiency
}

\author{
Lilliana Ciotta, Isabella Pagano, Vito Leanza, Mariagrazia Stracquadanio, Carlo Pafumi*
}

Institute of Obstetric and Gynecological Pathology, University of Catania, Catania, Italy; ${ }^{*}$ Corresponding Author: pafumi@unict.it

Received 14 March 2012; revised 16 April 2012; accepted 27 May 2012

\begin{abstract}
Premature Ovarian Failure (POF) is a heterogeneous syndrome characterized by Hypergonadotropic. Hypogonadism that affects about $1 \%$ of women younger than $\mathbf{4 0}$ years old, while an incidence of $1 / 1000$ is reported in women younger than 30 years old [1]. This hormonal disorder is caused by the loss of the ovarian response towards the pituitary gonadrotropins synthesis (FSH and LH) that are secreted according to the inhibitory feedback between hypothalamus, pituitary gland and gonads.
\end{abstract}

Keywords: Premature Ovarian Failure; Hypergonadotropic Hypogonadism; Inhibitory Feedback

\section{INTRODUCTION}

Premature Ovarian Failure (POF) is a heterogeneous syndrome characterized by Hypergonadotropic Hypogonadism that affects about $1 \%$ of women younger than 40 years old, while an incidence of $1 / 1000$ is reported in women younger than 30 years old [1].

This hormonal disorder is caused by the loss of the ovarian response towards the pituitary gonadrotropins synthesis (FSH and LH) that are secreted according to the inhibitory feedback between hypothalamus, pituitary gland and gonads.

Premature Ovarian Failure is characterized by the presence of amenorrhea before 40 years old, due to the cessation of the ovarian function (secondary amenorrhea) or to a primary ovarian defect with total absence of menarche (primary amenorrhea). The latter is the most severe form and in $50 \%$ of cases it is to refer to an ovarian dysgenesis $[2,3]$. The diagnosis is based on elevated levels of FSH (usually superior to $40 \mathrm{UI} / \mathrm{L}$ ), dosed twice consecutively with an interval of a few weeks from each other. Women affected by POF show anovulation and hypoestrogenism, primary or secondary amenorrhea, infertility, steroid hormones deficiency and elevated levels of gonadotropins.
The heterogeneity that underlies this syndrome reflects the multiplicity of possible causes that can determine it.

POF has a strong genetic component associated with an alteration of the genes mapping to the $\mathrm{X}$ chromosome, developing a primary role in the causes of ovarian dysgenesis $[4,5]$.

Despite the description of most candidate-genes, the causes of POF are still undetermined in most cases of Idiopathic forms $[6,7]$.

Different causes of POF are: Iatrogenic (surgery, chemotherapy, radiotherapy); Autoimmune, included the autoimmune polyglandular syndrome (APECED) caused by mutation of AIRE gene; Infections (CMV, Herpes Zoster); Chromosome $\mathrm{X}$ alterations (Turner syndrome, fragile X syndrome); Monogenetic defects: syndromic (syndrome by glycosylation defect of glycoprotein CDG-1, galactosemia, blepharophimosis epicanthus inversus syndrome BPES, pseudohypoparathyroidism type 1); isolated defects (mutation of FSH and LH receptors, FOXL2 mutation, BMP-15 mutation); Idiopathic [8].

The gene examined in our study is BMP-15.

The Bone Morphogenetic Proteins (BMPs) belong to the extracellular signaling-proteins that are part of Transforming Growth Factors-beta (TGF- $\beta$ ) superfamily, which include also growth and differentiation factors (GDFs). These proteins are synthesized as pre-propeptides, cleaved, and then processed to form dimeric proteins.

BMP-15 is a GDF oocyte-specific which stimulates the folliculogenesis and the granulose cells growth and it is expressed in oocytes during early folliculogenesis.

\section{MATERIALS AND METHODS}

In our study we performed the molecular analysis of BMP-15 gene in a small group of Sicilian patients affected by POF.

The study is a collaboration between the Section of Gynecologic Endocrinology, Institute of Obstetrics and Gynecology (S. Bambino Hospital, Catania), the Department of Biomedical Sciences and Department of Animal Biology of Catania, all structures belonging to the University of Catania.

This study examined a sample of women that, even if 
still limited, has been highly selected, focusing the attention on very young patients, in which the genetic causes truly have a major impact.

The control group consists of twenty young women in reproductive age, with regular cycles and not suffering from premature ovarian failure or other pathological conditions. This select group is homogeneous for age.

$\mathrm{X}_{2}$ analysis was used to compare the data of POF and control groups.

Diagnostic criteria considered for the recruitment of these patients, were extremely selective in order to exclude in advance cases of ovarian insufficiency due to other causes (Tables 1 and 2).

The criteria for inclusion in the study were the followings (Table 1):

1) Primary amenorrhea and/or secondary amenorrhea (spontaneous absence of menstruation for at least 12 months);

2) Patients younger than 35 years old (Average Age is 26.6 years, $\min =14$ years, $\max =40$ );

3) Average Levels of serum FSH equal or superior at $50 \mathrm{mUI} / \mathrm{mL}$ and average serum levels of estradiol less than $30 \mathrm{pg} / \mathrm{mL}$ in, at least, three different dosages;

4) Absence of concomitant endocrine and/or autoimmune disorders.

Each patient and control were assessed clinically, with a complete medical and gynecological history, including the history of menses, age at menopause, LH and FSH levels (two times at one-month intervals), and pelvic ultrasound. Patients with associated endocrinopathies, autoimmune disorders, iatrogenic agent such as pelvic surgery, chemotherapy, and radiotherapy, and infections, were excluded. Karyotyping to check for chromosomal anomalies was performed in all patients and controls. Those with abnormalities were excluded from the study.

All the patients enrolled had a normal karyotype, 46 $\mathrm{XX}$, and they did not suffer from any autoimmune disorders: these were all necessary conditions to reduce the possibility that the premature ovarian failure could be correlated to different causes from those genetic.

Three of the twenty patients examined were affected by primary amenorrhea, while the remaining by seconddary amenorrhea; furthermore, every patient showed high levels of pituitary gonadotropins (FSH > $50 \mathrm{mUI} / \mathrm{ml}$ )

Table 1. Criteria for inclusion in the study $(n=20)$.

\begin{tabular}{lc}
\hline \multicolumn{1}{c}{ Average age } & 26.6 years $(14-40)$ \\
\hline Average level FSH serum & $>50 \mathrm{mUI} / \mathrm{ml}$ \\
Average level estradiol serum & $<30 \mathrm{pg} / \mathrm{ml}$ \\
Primary or secondary amenorrhea & $(18 \pm 4)$ months \\
Absence of concomitant autoimmune diseases & \\
\hline
\end{tabular}

Table 2. Characteristics of patients recruited in the study (n = 20).

\begin{tabular}{|c|c|c|c|c|}
\hline N. & Patients & Age & FSH (UI/l) & Polymorphism \\
\hline 1 & B.A. & 20 & 58 & \\
\hline 2 & P.L. & 40 & 67 & \\
\hline 3 & B.L. & 40 & 53 & \\
\hline 4 & F.S. & 27 & 56 & $\begin{array}{c}308 \mathrm{~A}>\mathrm{G} \\
\text { exon1 }\end{array}$ \\
\hline 5 & S.V. & 20 & 60 & \\
\hline 6 & P.P. & 34 & 55 & \\
\hline 7 & S.M. & 35 & 84 & \\
\hline 8 & B.L. & $\begin{array}{l}15 \text { (primary } \\
\text { amenorrhea) }\end{array}$ & 40 & \\
\hline 9 & F.C. & $\begin{array}{l}18 \text { (primary } \\
\text { amenorrhea) }\end{array}$ & $>100$ & \\
\hline 10 & R.R. & 25 & 60 & \\
\hline 11 & V.A. & 26 & $>100$ & $\begin{array}{c}308 \mathrm{~A}>\mathrm{G} \\
\text { exon1 }\end{array}$ \\
\hline 12 & G.S. & 18 & $>100$ & \\
\hline 13 & G.L. & $\begin{array}{l}14 \text { (primary } \\
\text { amenorrhea) }\end{array}$ & 92 & \\
\hline 14 & D.G. & 23 & 65 & \\
\hline 15 & R.V. & 17 & 51 & $\begin{array}{l}788 \text { TCT } 789 \\
\text { exon } 2\end{array}$ \\
\hline 16 & M.A. & 25 & 45 & \\
\hline 17 & I.I. & 26 & 57 & \\
\hline 18 & P.I. & 33 & 55 & \\
\hline 19 & A.L. & 38 & 63 & \\
\hline 20 & P.S. & 38 & 72 & \\
\hline
\end{tabular}

and low estrogen levels $(<30 \mathrm{pg} / \mathrm{ml})$.

Their average age was 27 years old and five of them were under 20; the importance of age is explained by the fact that in younger patients the genetic component plays a major role in the demonstration of pathology.

The aim of cytogenetic analysis is to identify numerical and/or structural chromosomal abnormalities. Therefore, considering that chromosomal abnormalities have strong implications on the onset of POF, the preliminary confirmation of a normal karyotype results to be a further selective diagnostic criteria, that allows to exclude the chromosomal defects as a cause of ovarian failure.

The BMP-15 gene maps to the short arm of the $\mathrm{X}$ chromosome in Xp11.2 position, inside one of the critical areas of POI. The gene codifies for BMP-15 protein and it consists of two exons: the first exon codifies 17 amino acids of signal-peptide and the first part of the 
pre-peptide, while the second encodes for the remaining area of signaling pre-peptide and for the whole mature domain formed by 125 amino acids [9].

Indeed, as the other proteins of the TGF- $\beta$ superfamily, BMP-15 is synthesized primarily as an inactive precursor and, only after the removal of signal peptide, generates the proprotein that binds covalently to another identical protein, forming the homodimers, or to GDF-9 protein, determining, instead, the formation of heterodimers. These dimers are then cleaved by specific endoproteases at the pro-region level forming mature peptides. These are mature peptides that, binding to the specific receptors exposed on the granulosa cells, activate the cascade of Smad 1/5/8 signal proteins. Infact BMP-15, as a ligand, activates its own type II receptor, BMPR-2, which interacts with the type I receptor, ALK-6 activated, responsible for the beginning of intracellular cascade, operated by Smad complex [10].

The BMP-15 protein is primarily expressed in oocytes, from the primary follicle stage until ovulation. Therefore, considering its close correlation with the ovarian function, all the studies conducted on this gene showed, after the identification of its mutations in patients affected by premature ovarian insufficiency, that BMP-15 is one of the responsible genes for this heterogeneous disorder. The Human Growth Differentiation Factor 9 (GDF-9), such as BMP-15, is another member of the TGF- $\beta$ superfamily. This gene maps to the long arm of chromosome 5 (5q31.1). Although located on different chromosomes, GDF-9 and BMP-15 are two very similar genes that share similar structural and functional characteristics; both encoded proteins are able to form heterodimers and correlate with ovarian failure. It is demonstrated that women with ovarian dysfunction (particularly, follicular dysfunction), show mutations of this gene and so, in the same way of BMP-15, it is a cause of POF.

The mutation analysis obtained by sequencing the exons of the BMP-15 gene enables us to:

1) Identify the presence of mutations and thus to confirm the implication of the gene in the etiopathogenesis of premature ovarian failure;

2) Try to identify a genotype-phenotype correlation in relationship to the founder mutations.

The evaluation of the impact of this gene in cases of familiar POF could allow an early diagnosis of mutation in relatives of patients that are not affected yet, informing them precociously on the future risks of the disease development, in order to plan better their reproductive choices.

For the study of the patients' karyotype has been done an external heparinized blood collection, from which have been prepared lymphocytes cultures on dedicated grounds, afterwards they have been processed through centrifugation and fixative addition in order to perform the slides scratch. To be able to study the chromosomes in a proper way, we need to spread them on the plate in order to be recognized in their entirety. It's necessary an optimal temperature of $20^{\circ} \mathrm{C}$ with a relative humidity of $50 \%-60 \%$. Two or three days must pass before the banding of the slides. The slides prepared and coloured are later observed under microscope. For the genomic DNA extraction, the erythrocytes were submitted to osmotic lysis in order to eliminate the hemoglobin, and the leucocytes nucleus were recovered, and later were lysed to permit the genomic Dna release, that was subsequently purified and quantized.

To be able to analyze the BMP-15 exons we proceeded with their successive amplification through PCR, using specific primers. To consider optimal a couple of primers the fundamental requirement is the ability to hybridize efficiently and specifically the sequence. The two primers have a distance flexible that could differ from 100 to 10,000 base pairs (10 KPB). Preferably the length of the primers should be composed by 20 and 30 bases. The nucleotide sequence of the primers is determined according to the DNA sequence, through the use of specific software. In our laboratory we use the software "primer v. 1.1" which analyzes the different sections of DNA, delete stretches of DNA rich in GC and repetitions of bases or base groups, too. The software also allows to identify and discard the oligonucleotides that can fold back to form hairpin or those which tend to form dimers or trimers. The exons, amplified through PCR starting from the genomic DNA of the patients, have been sequenced using the Sanger method, applied to an automatic sequencer. The sequencing allows to obtain the DNA sequence, that is the exact order of the nucleotides that comprise it. The Sanger method consists in using nucleotides, that modified in their chemical structure (ddNTPs) and labeled with fluorochrome (BigDye Terminetor) are incorporated in the synthesis of neo-filament from a heat-resistant DNA polymerase, which uses as a trigger one of the two specific primers for each exon amplified.

The dideossinucleotiditrifosfato are chain terminators, in fact, do not have the 3'-OH on the C3' of the ribose, and then once inserted on the nascent strand, blocking the reaction of Taq polymerase in that, the lack of the hydroxyl group, does not allow the formation of the phosphodiester bond 3' - 5'. In this way we obtain fluorescent complementary strands, each of different length, ending with one of the four ddNTPs to 3' end. These labeled fragments are separated by capillary electrophoresis on a polyacrylamide polymer. During the electrophoresis, the sample is hit by a light source (laser) that excites the fluorophores with different emission of fluorescence that is detected by a sensor. Finally, thanks to a specific software, the signal is processed in the form of 
electro pherogram.

\section{RESULTS}

The study conducted on our twenty Sicilian women affected by POF, apparently idiopathic, did not identify any mutation of the gene responsible for the pathology.

However, in the POF group have been found 3 polyformisms, unlike the control group where the incidence has been of only a single case.

Infact, $A / G$ heterozygosity in position 308 of the cDNA (c.308 A > G), located in the exon 1 of the BMP15 gene, although determining an amino acid change (p.Asn 103 ser), is also found in normal subjects (control group) as well as in the cases.

The second polymorphism is to be referred to an inclusion in the fragment 2.2 of exon 2 of BMP-15 gene (778 TCT 789), also present in the control group.

Through the $\mathrm{X}^{2}$ analysis has not been found a statistically significant difference between the two groups $(\mathrm{P}<$ $0.59)$.

Moreover, from the cytogenetic survey had emerged that both women were karyotype normal (46, XX) and so this excluded the hypothesis that the disease was caused by a Chromosome $\mathrm{X}$ defect, validating the idea that it could be a gene defect.

In the Electropherogram obtained by automatic sequencing $\mathrm{ABI} 310$, we can observe, for example, that in patient N.4 the polymorphic variant, in heterozigosity, c.308A > G, it's responsible of the substitution of a serine with an asparagine in position 103.

\section{DISCUSSION}

The menopausal period represents the end of woman's procreative capacity. Actually, it has been well-known for a long time that the decline of ovarian function begins about 10 years before the appearance and the establishing of a clear menopause [11].

This means that 10 years before the spontaneous cessation of menstrual cycle, even in the presence of ovulatory-type cycles, the capacity of oocyte to be fertilized decreases significantly, as a result of the inevitable phenomena of cellular aging. Statistically, in a woman destined to go into menopause in a regular age range (48 54 years old), her reproductive potential decreases already from 38 - 40 years old, in a way positively related to the chronological age increase. This is usually highlighted, from a laboratory point of view, by a progressive increase of serum FSH levels, which, if equal or superior to 15 - $20 \mathrm{mUI} / \mathrm{mL}$ in the follicular phase of the cycle, and if associated to low levels of estradiol, clearly denotes the decline of ovarian reserve [12].

In IVF Centers, infact, the pregnancy rates are signifycantly reduced in presence of FSH levels equal or supe- rior to $20 \mathrm{mUI} / \mathrm{mL}$. The most precocious markers of reduced ovarian reserve are represented by the Inhibin B and, above all, Müllerian inhibitory factor [13].

The transition from normal intraovarian peptides values appears to be earlier than serum FSH and E2 levels. However, various clinical and laboratory trials [14] have recently underlined that also the valuation of these peptides is not able to certainly anticipate future decline of ovarian reserve, as, similarly to what has been shown in FSH and E2 levels, the modification of Müllerian inhibitory factor levels and, specially, of Inhibin B is associated with an already concomitant and inexorable reduction of the ovarian reserve [15].

The very precocious identification of loss of ovarian reproductive ability is very important in cases of premature or precocious menopause (POF): in this case, indeed, it is very important to clearly provide the patient with the "window" of reproductive potential of which she can take advantage.

Nowadays, the hormone replacement therapy (HRT) has reached an absolute perfection and effectiveness.

Nowadays, in fact the HRT enables a woman affected by precocious menopause, to limit, or even to reset, the potential and serious consequences that she would face in presence of 10 - 20 years premature menopause[16]: vasomotor symptoms, depression, osteoporosis, genitourinary atrophy, dysmetabolism, dermal collagen reducetion, increase of vascular diseases.

The issues related to the premature exhaustion of ovarian function are also extended to lipid metabolism, with tendency to increase the average total cholesterol and LDL-cholesterol levels, and with a reduction in HDL cholesterol fraction [17].

The dysmetabolic lipid manifestations can associate with a mean blood pressure values modifications (both systolic and diastolic), with modifications represented by a tendency to the constant increase of values and by occasional blood pressure-raising phenomena.

The lipid dismetabolism and the early modification of blood pressure parameters involve a higher and/or early risk of athero-sclerotic vascular diseases at cardiac, cerebral and aortic level [16], with a significant reduction in global quality-of-life. The reduction of individual psychophysical performances is also consequent on the ponderal growth (more or less substantial according to family characteristics and/or basal metabolism) that the cessation of ovarian function involves. As a matter of fact, the average energetic expenditure consequent to ovarian activity during the fertile period of the female life is approximately equal to $300 \mathrm{kcal} / \mathrm{die}$; consequently, even without increasing the daily caloric intake, the cessation of ovarian function is associated with an increased weight gain tendency.

From an etiopathogenetic perspective, an early deple- 
tion of ovarian function is usually the result of autoimmune processes (with the formation of anti-ovarian antibodies and perifollicular lymphocytic infiltrates), chromosomal alterations (mainly with features of mosaicism), or a gonadal damage following chemotherapy or radiation treatment protocols.

The issues related to profound hypoestrogenemic state and to the subjective and metabolic consequences can be easily overcome by the hormone replacement therapy, that, if established with appropriate timing and mode of administration, is able to prevent entirely (eminently in a symptomatic side) the almost inevitable subjective and objective consequences that premature cessation of ovarian function involves [18].

Thus, the HRT, can overcome and solve clinical metabolic and organ problems related to premature depletion of ovarian function [19].

Unfortunately, the question related to reproductive capacity is very different. The rising levels of FSH and E2 are usually associated with failure of all IVF methods. Also the evaluation of the NHI and/or the Inhibin2 are often unable and/or inadequate to "estimate" adequately, with predetermined times, the margin of residual reproductive capacity. It seems, therefore, very interesting the opportunity to identify an additional methodology, suitable for early identification of the so-called "subjects at risk", that are women with increased possibility of encountering the collapse of the early ovarian function. Particularly, subjects at risk can be considered all young women with a first or second-degree relatives with POF, considering the high incidence of POF in established relatives' groups, even in absence of specific chromosomal abnormalities [20].

The evaluation of the chromosomal map is generally negative in relatives of women affected by POF, resulting normal in the great majority of cases. Instead, it is possible to identify early the subjects at risk through a more detailed genetic evaluation.

In our case, finding no mutation can be justified:

- by the fact that the small number of the examined samples is not statistically significant;

- the Sicilian population is not predisposed to a mutation of BMP-15 gene;

- in these women the cause of premature ovarian failure is determined by other genes responsible for POF.

From our study has emerged that the polymorphisms presence in the selected cases has a 15\% percentage, while such polymorphisms in the control group have shown themselves with a lower frequency (5\%).

The detected polymorphic variant has been described in the literature as a responsible, in Caucasian women, of $9.8 \%$ of cases of POF, of $10 \%$ of women with primary amenorrhea and none of the patients affected by seconddary amenorrhea. Instead, in the control-group this po- lymorphic variant has a frequency of 5\%, demonstrating that this variant is mainly present in cases of ovarian failure and of primary amenorrhea rather than in patients-control. This has led us to think that the c.308 A > $\mathrm{G}$ and 788 TCT 789 polymorphisms may be correlated with POF [21-25].

In agreement with the data of literature we can confirm that such polymorphisms occur more frequently in the cases of premature ovarian failure, rather than in the group of control, confirming the implication that this gene could have in the manifestation of the pathology.

With the intention to continue recruiting highly selected patients affected by POF, we aim to continue to sequence BMP-15 gene, in order to identify its mutations and, in case, to extend this research also towards other genes responsible for premature ovarian failure. Taken together, these studies could contribute to clarify the knowledge concerning ovarian and folliculogenesis development and, possibly, in the future to elaborate the molecular tests able to recognize the women at risk of developing POF.

\section{ACKNOWLEDGEMENTS}

Valentina Pafumi has carried out English language editing for this article.

\section{REFERENCES}

[1] Persani, L., Rossetti, R., Cacciatore, C. and Bonomi, M. (2009) Primary ovarian insufficiency: X chromosome defects and autoimmunity. Journal of Autoimmunity, 33, 35-41. doi:10.1016/j.jaut.2009.03.004

[2] Santoro, N. (2003) Mechanism of premature ovarian failure. Annales d Endocrinologie, 64, 87-92.

[3] Timmreck, L.S. and Reindollar, R.H. (2003) Contemporary issues in primary amenorrhea. Obstetrics and Gynecology Clinics of North America, 30, 287-302. doi:10.1016/S0889-8545(03)00027-5

[4] Simpson, J.L. and Rajkovic, A. (1999) Ovarian differentiation and gonadal failure. American Journal of Medical Genetics, 89, 186-200. doi:10.1002/(SICI)1096-8628(19991229)89:4<186::AIDAJMG3>3.0.CO;2-5

[5] Goswami, D. and Conway, G.S. (2005) Premature ovarian failure. Human Reproduction Update, 11, 391-410. doi:10.1093/humupd/dmi012

[6] Acherman, J.C., Ozisik, G., Meeks, J.J. and Jameson, J.L. (2002) Perspective: Genetic causes of human reproductive diseases. Journal of Clinical Endocrinology \& Metabolism, 87, 2447-2454. doi:10.1210/jc.87.6.2447

[7] Shimasaki, S., Moore, R.K., Otsuka, F. and Erickson, G.F. (2004) The bone morphogenetic protein system in mammalian reproduction. Endocrine Reviews, 25, 72-101. doi:10.1210/er.2003-0007

[8] Beck-Peccoz, P. and Persani, L. (2006) Premature ovar- 
ian failure. Orphanet Journal of Rare Diseases, 1, 9.

[9] Dube, J.L., Wang, P., Elvin, J., Lyons, K.M., et al. (1998) The bone morphogenetic proteins 15 gene is X-linked and expresses in oocytes. Molecular Endocrinology, 12, 18091817. doi:10.1210/me.12.12.1809

[10] Moore, R.K., Otsuka, F. and Shimasaki, S. (2003) Molecular basis of bone morphogenetic protein-15 segnaling in granulosa cells. Journal of Biological Chemistry, 278, 304-310. doi:10.1074/jbc.M207362200

[11] Wallace, W.H. and Kelsey, T.W. (2010) Human ovarian reserve from conception to the menopause. PLoS ONE, 5, e8772. doi:10.1371/journal.pone.0008772

[12] Su, H.I. and Freeman, E.W. (2009) Hormone changes associated with the menopausal transition. Minerva Ginecologica, 61, 483-489.

[13] Durlinger, A.L., Visser, J.A. and Themmen, A.P. (2002) Regulation of ovarian function: The role of anti-Mullerian hormone. Reproduction, 124, 601-609. doi:10.1530/rep.0.1240601

[14] Massin, N., Gougeon, A., Meduri, G., Thibaud, E., et al. (2004) Significance 123 of ovarian histology in the management of patients presenting a premature ovarian failure. Human Reproduction, 19, 2555-2560. doi:10.1093/humrep/deh461

[15] Chand, A.L., Harrison, C.A. and Shelling. A.N. (2010) Inhibin and premature ovarian failure. Human Reproduction Update, 16, 39-50. doi:10.1093/humupd/dmp031

[16] Henderson, B.E., Paganini-Hill, A. and Ross, O.K. (1991) Decreased mortality in users of estrogen replacement therapy. Archives of Internal Medicine, 151, 75-78. doi:10.1001/archinte.1991.00400010095012

[17] Sznajderman, M. and Oliver, M.F. (1963) Spontaneous premature menopause, ischaemic heart-disease and serum lipids. Lancet, 281, 962-965. doi:10.1016/S0140-6736(63)91747-1

[18] Walsh, B.W., Schiff, I., Rosner, B. et al. (1991) Effect of postmenopausal estrogen replacement on the concentrations and metabolism of plasma lipoproteins. New England Journal of Medicine, 325, 1196-1204. doi:10.1056/NEJM199110243251702

[19] Samsioe, G. (1992) Effects of hormone replacement therapy on lipid metabolism and incidence of cardiovascular disease. In: Samsioe, G., Ed., Cardiovascular disease and HRT. New perspectives. Parthenon Publishing Group, Carnforth, 35-40.

[20] Ciotta, L., Carco, C., Di Grazia, S., Marletta, E., et al. (1996) Ruolo della terapia ormonale sostitutiva in donne affette da menopausa precoce. Contraccezione Fertilità Sessualità, 23, 68-71.

[21] Van Kasteren, Y. (2001) Treatment concepts for premature ovarian failure. Journal of the Society for Gynecologic Investigation, 8, S58-S59.

[22] Ciotta, L., Pagano, I., Stracquadanio, M., Pafumi, C., Teodoro, M.C. and Giuffrida, E. (2010) Innovative genetic, clinical and endocrinological aspects in young females affected with premature ovarian insufficiency. Minerva Ginecologica, 62, 501-507.

[23] Rossetti, R., Di Pasquale, E., Marozzi, A., Bione, S., Toniolo, D., Grammatico, P., Nelson, L.M., Beck-Peccoz, P. and Persani, L. (2009) BMP15 mutations associated with primary ovarian insufficiency cause a defective production of bioactive protein. Human Mutation, 30, 804810. doi:10.1002/humu.20961

[24] Inagaki, K. and Shimasaki, S. (2010) Impaired production of BMP-15 and GDF-9 mature proteins derived from proproteins with mutations in the proregion. Molecular and Cellular Endocrinology, 328, 1-7. doi:10.1016/j.mce.2010.05.017

[25] Simpson, J.L. (2008) Genetic and phenotypic heterogeneity in ovarian failure: Overview of selected candidate genes. Annals of the New York Academy of Sciences, 1135, 146-154. doi:10.1196/annals.1429.019 